\title{
Toll-like receptor 9, a possible blocker of non-alcoholic steatohepatitis?
}

\author{
Soung Won Jeong \\ Division of Gastroenterology and Hepatology, Department of Internal Medicine, Soonchunhyang University Seoul Hospital, Soonchun-
} hyang University College of Medicine, Seoul, Korea

Keywords: Non-alcoholic fatty liver disease; Toll-like receptor 9; Immune system; Interferon-gamma; T-cell

\section{See Article on Page 216}

Toll-like receptor 9 (TLR9) is a transmembrane protein which is expressed on the cell surface of immune system cells such as T cells, macrophages, natural killer cells, and other antigen-presenting cells. ${ }^{1}$ TLR9 binds the cytosine-phosphate-guanine dideoxynucleotide motif within bacterial and viral DNA, and triggers signaling cascades that lead to a pro-inflammatory cytokine response, including interferon $\gamma$ (IFN- $\gamma)$, tumor necrosis factor a (TNF-a), and interleukin 6 (IL-6). ${ }^{2,3}$ Plasma from mice and patients with non-alcoholic steatohepatitis (NASH) contained high levels of mitochondrial DNA (mtDNA) from microparticles of hepatocyte origin and activated TLR9. ${ }^{4}$ TLR9 ligands, such as mtDNA, may activate the innate immune system through TLR9, which leads to the development of steatohepatitis, fibrosis, and insulin resistance. ${ }^{5}$

The prevalence of nonalcoholic fatty liver disease (NAFLD) increases worldwide, and it is emerging as a major cause of chronic liver disease. Among the various phenotypes of NAFLD, NASH is highly likely to progress to the development of end-stage liver dis- ease and cardiometabolic disease, resulting in both liver-related and non-liver-related mortality.

In this issue of Clinical and Molecular Hepatology, Alegre et al. ${ }^{8}$ reported that TLR9 expression on liver and peripheral T cells was the lowest in patients with simple steatosis (SS) and was positively associated with anthropometric, biochemical, and histopathological features of NAFLD. The authors interpreted the mechanism of an overall downregulation of TLR9 on T cells from SS patients as a protective adaptation from hepatocellular injury, whereas a constant, unvarying expression in patients with NASH was considered as a failure of this regulatory mechanism.

At baseline, patients with SS showed a low frequency of circulating type $1 \mathrm{CD}^{+}$cells. In vitro co-stimulation of $\mathrm{T}$ cells in these patients induced a limited number of IFN- - -producing $C D 8^{+} T$ cells. However, NASH patients showed a higher frequency of IFN- $\gamma$ producing cells compared to SS patients and controls at baseline. These results confirmed the previous data concerning a synergism between $T$ cell receptors and TLR9 during the induction of IFN- ${ }^{9}{ }^{9}$

IFN- $\gamma$ is produced predominantly by natural killer cells as part of

\section{Abbreviations:}

IFN- $\gamma$, interferon $\gamma$; IL-6, interleukin 6; mtDNA, mitochondrial DNA; NAFLD, nonalcoholic fatty liver disease; NASH, non-alcoholic steatohepatitis; SS, simple steatosis; TLR9, toll-like receptor 9; TNF-a, tumor necrosis factor a

\section{Corresponding author: Soung Won Jeong}

Division of Gastroenterology and Hepatology, Department of Internal Medicine, Soonchunhyang University Seoul Hospital, Soonchunhyang University College of Medicine, 59 Daesagwan-ro, Yongsan-gu, Seoul 04401, Korea

Tel: +82-2-710-3076, Fax: +82-2-709-9696

E-mail: jeongsw@schmc.ac.kr

https://orcid.org/0000-0003-2855-6011 
the innate immune response, and by $\mathrm{CD} 4^{+} \mathrm{T}$ helper cells and $\mathrm{CD} 8^{+}$ cytotoxic T cells once antigen-specific immunity develops. It is an important activator of M1 macrophages (classically activated macrophages), which play an important role as the pro-inflammatory type in phagocytosis and secretion of pro-inflammatory cytokines. Inflammation driven by M1 macrophages is counterbalanced by alternatively polarized M2 macrophages that promote the resolution of inflammation and tissue repair. ${ }^{10,11}$ Wan et al. ${ }^{12}$ suggested that promoting M2-induced M1 Kupffer cell apoptosis might prove to be a relevant strategy to limit high fat-induced inflammation and hepatocyte injury.

Innate immune signaling has been considered to play a key role in initiating and developing hepatic inflammation, contributing to the transition from nonalcoholic fatty liver to NASH. Recently, Garcia-Martinez et al. ${ }^{4}$ tried TLR7/9 antagonist IRS954 to block the ability of hepatocyte mtDNA from high fat diet fed mice to activate proinflammatory cytokines in primary macrophages. IRS954 led to a significant reduction in histological NAFLD parameters, including steatosis, ballooning, and inflammation, and also reduced pro-IL-1 $\beta$, IL-6, TNF- $\alpha$, and serum alanine aminotransferase levels.

In summary, intrahepatic and peripheral TLR9 protein expressions were the lowest in patients with SS, which was likely a protective adaptation from hepatocellular injury. On the other hand, the constant expression in patients with NASH may be a failure of this regulatory mechanism. However, NASH patients showed a higher frequency of IFN- $\gamma$ producing cells compared to SS patients and controls at baseline. TLR9 activation initiates the innate immune system, which leads to the development of steatohepatitis, fibrosis, and insulin resistance.

Blocking TLR9 may reverse NASH and be a possible therapeutic target for the blocking of NASH. Further study is needed to examine the effect of TLR9 ablation and TLR9 antagonism on T cells, as well as to investigate the role of TLR9 in the activation of stellate cells and fibrosis.

\section{Acknowledgements}

This study was supported by the Soonchunhyang University Research Fund.

\section{Conflicts of Interest}

The author has no conflicts to disclose.

\section{REFERENCES}

1. Du X, Poltorak A, Wei Y, Beutler B. Three novel mammalian toll-like receptors: gene structure, expression, and evolution. Eur Cytokine Netw 2000;11:362-371.

2. Bauer $S$, Kirschning CJ, Häcker $H$, Redecke V, Hausmann S, Akira $S$, et al. Human TLR9 confers responsiveness to bacterial DNA via species-specific CpG motif recognition. Proc Natl Acad Sci U S A 2001;98:9237-9242.

3. Hemmi H, Takeuchi O, Kawai T, Kaisho T, Sato S, Sanjo H, et al. A Toll-like receptor recognizes bacterial DNA. Nature 2000;408:740745.

4. Garcia-Martinez I, Santoro N, Chen Y, Hoque R, Ouyang X, Caprio $S$, et al. Hepatocyte mitochondrial DNA drives nonalcoholic steatohepatitis by activation of TLR9. J Clin Invest 2016;126:859-864.

5. Miura K, Kodama Y, Inokuchi S, Schnabl B, Aoyama T, Ohnishi H, et al. Toll-like receptor 9 promotes steatohepatitis by induction of interleukin-1beta in mice. Gastroenterology 2010;139:323-334.e7.

6. Yoo JJ, Kim W, Kim MY, Jun DW, Kim SG, Yeon JE, et al. Recent research trends and updates on nonalcoholic fatty liver disease. Clin Mol Hepatol 2019;25:1-11.

7. Lee YH, Cho Y, Lee BW, Park CY, Lee DH, Cha BS, et al. Nonalcoholic fatty liver disease in diabetes. Part I: epidemiology and diagnosis. Diabetes Metab J 2019;43:31-45.

8. Alegre NS, Garcia CC, Billordo LA, Ameigeiras B, Poncino D, Benavides J, et al. Limited expression of TLR9 on T cells and its functional consequences in patients with nonalcoholic fatty liver disease. Clin Mol Hepatol 2020;26:216-226.

9. Kranzer K, Bauer M, Lipford GB, Heeg K, Wagner H, Lang R. CpGoligodeoxynucleotides enhance $\mathrm{T}$-cell receptor-triggered interferongamma production and up-regulation of CD69 via induction of antigen-presenting cell-derived interferon type I and interleukin-12. Immunology 2000;99:170-178.

10. Murray PJ, Wynn TA. Protective and pathogenic functions of macrophage subsets. Nat Rev Immunol 2011;11:723-737.

11. Sica A, Mantovani A. Macrophage plasticity and polarization: in vivo veritas. J Clin Invest 2012;122:787-795.

12. Wan J, Benkdane M, Teixeira-Clerc F, Bonnafous S, Louvet A, Lafdil F, et al. M2 Kupffer cells promote M1 Kupffer cell apoptosis: a protective mechanism against alcoholic and nonalcoholic fatty liver disease. Hepatology 2014;59:130-142. 